\title{
Fantômes et contrefaçons dans l'œuvre de Béroalde de Verville : ouvrages virtuels, fictifs et fictionnels ${ }^{1}$
}

GILLES POLIZZI

Université de Haute Alsace-Mulhouse

This article proposes to take inventory of and examine, in the abundant vervilienne production, the absent works, non-existent or "disguised." Reflecting upon the relationship between title and identity, as well as our aptitude to deduce from a title the content and character of a work, the author of this article defines the process of "bibliographical illusion," used frequently by Verville, who, in his bibliography, multiplies these ghosts. Hence, this article sorts the works while considering, in an approximately chronological order, virtual books (announced, but not published and perhaps not written), fictitious books (those whose title suggests camouflage or forgery), and invented disguises; because the science of the book is fueled by invention. This article concludes with the unexpected restoration of a book as real, from the category of fictitious works. Bibliography is then not only the art of listing titles, but also the art and the science of linking titles with works the art of testing the game of bookish inventions and trickery.

$\mathrm{O}$ $\mathrm{n}$ se propose de recenser et d'examiner, dans l'abondante production vervilienne, les œuvres absentes, inexistantes ou « déguisées », et qui nous semblent trop nombreuses pour que leur cas soit fortuit. C'est le prétexte à une réflexion sur la relation entre intitulé et identité sur laquelle repose leur virtualité. Il y a en effet dans notre aptitude à déduire d'un titre le contenu et le caractère d'une œuvre, le ressort d'une "illusion bibliographique » dont Verville use et abuse, ce qui a pour effet de multiplier les "fantômes » dans sa bibliographie virtuelle. On opèrera un tri, en considérant, dans un ordre approximativement chronologique, les livres virtuels (annoncés mais non publiés et peut-être non écrits), les livres fictifs (ceux dont le titre annonce un déguisement ou une forgerie), et les déguisements fictionnels; enfin, puisque 
la science du livre se nourrit d'inventions, on conclura par un livre impossible, parce ce que Henri-Jean Martin en personne donne d'excellentes raisons pour qu'il n' existe pas : on atteste pourtant l'avoir tenu entre nos mains.

Saluons, au seuil de cette étude, le beau travail bibliographique accompli par Véronique Luzel, l'un des meilleurs sur le sujet, dans une thèse, dont l'intérêt dépasse le propos annoncé, à savoir l'édition critique du Palais des curieux ${ }^{2}$ de Béroalde de Verville. Si cette thèse n'a pas suscité notre enquête, entreprise longtemps auparavant, en vue d'une bibliographie critique de Verville annoncée dans la collection Memini mais restée en suspend, elle y aura mis fin. Et quoiqu' on lui conteste des "fantômes » trop généreusement admis dans sa bibliographie, on avoue volontiers que son travail aura rendu possible le nôtre. On souhaite donc lui dire la franche admiration que nous inspire sa science du désordre vervilien. Mais venons-en à notre propos qu'on introduira par cette réflexion de Giorgio Agamben sur les œuvres-fantômes :

Toute ouvre écrite peut être considérée comme le prologue ou plutôt le moule à cire perdue d'une ceuvre jamais rédigée et destinée à ne jamais l'être, parce que les œuvres ultérieures, elles-mêmes préludes ou moulages d'autres œuvres absentes ne représentent que des esquisses ou des masques mortuaires. L'ouvre absente, bien qu' on ne puisse la situer exactement dans une chronologie, constitue ainsi les œuvres écrites en prolegomena ou en paralipomena d'un texte inexistant; ou bien de manière plus générale en parerga qui ne trouvent leur vrai sens qu'en regard d'un ergon illisible. Selon la belle image de Montaigne, elles sont les grotesques encadrant un portrait jamais peint ; ou suivant l'intention d'une lettre pseudo-platonicienne, les contrefaçons d'un écrit impossible. ${ }^{3}$

«Contrefaçons d'un écrit impossible » : voilà qui s'applique bien à la prolifération des œuvres « absentes » chez Verville. Parmi les 37 (si notre compte est bon) que lui prête trop généreusement Véronique Luzel, 13 sont perdues ou n'ont jamais été écrites ${ }^{4}$. Il n'est pas non plus hors-sujet de préciser que l'œuvre nonécrite dont parlait Agamben, projet d'une « œuvre obstinément restée dans un tiroir » est un essai intitulé La Voce humana, dont l'auteur ne donne que cet incipit : 
existe-t-il une voix humaine, une voix qui soit la voix de l'homme comme la stridulation est la voie de la cigale (...) et si elle existe, le langage est-il cette voix $?^{5}$

Car cette conception de l'œuvre désespérément in progress, en ce qu'elle situe l'inachèvement au cœur du texte, retentit sur les définitions respectives del'œuvre et du livre : à partir de quand faut-il considérer que l'oeuvre existe? Au moment où s'achève - mais comment savoir que c'est le cas? - une rédaction qui n'est déjà plus conforme à la virtualité d'un projet, ou bien lors de sa publication ? La seconde solution a l'avantage de produire des certitudes ; c'est pourquoi elle est communément adoptée quoiqu'elle méconnaisse les «brouillons » et autres variantes que la critique génétique nomme depuis deux décennies les « avanttextes » de l'œuvre. Ou bien encore, au moment où le manuscrit, supposé achevé, est communiqué (particulièrement aux bibliographes toujours avides d'inédits susceptibles de devenir leur invention ${ }^{6}$ ), celui où l'œuvre, non moins hypothétique que Les Fruits d'or de Nathalie Sarraute, vient au monde parce qu'elle est parlée (puisqu'on en parle) aussi bien qu'entendue comme une voix autonome? Point de vue très incommode, mais qui s'applique particulièrement à la fiction du Moyen de parvenir de Verville; nous y reviendrons donc.

Pour l'heure attachons-nous au problème du dénombrement, compliqué par la manifestation de ces absentes, peut-être seulement virtuelles et qu'il faut pourtant prendre en compte et tenter d'insérer dans la chronologie des éditions. Le problème ne tient pas à cette virtualité absolue que postule Agamben et que partagent tous les auteurs, mais à un artifice propre à Verville. Dès l'origine, dans l'édition de ses premiers recueils, il brouille sciemment les repères afin de donner l'impression qu'il est déjà à la tête d'une œuvre pléthorique ; cette illusion référentielle au sens bibliographique du terme (lequel consiste à postuler l'existence d'une œuvre à partir de sa référence) résulte d'une mise en scène éditoriale qui nous semble une nouveauté dans le cours de l'histoire du livre : voyons en les procédés.

\section{L’illusion référentielle : l'exemple des Appréhensions spirituelles}

Dès 1583, au temps de ses premières publications sous son nom d'auteur, Verville conçoit une stratégie de la fragmentation de l'œuvre. Elle produit un recueil qui, en apparence tout au moins, contient des livres. Pour faire ressortir le 
procédé, on réservera les italiques aux titres des œuvres effectivement publiées, en soulignant les syntagmes qui désignent, pourraient désigner, ou désigneront plus tard, des œuvres autonomes. On indique par ailleurs en caractères gras les termes des paratextes qui réfèrent à un apparat éditorial par la désignation d'une forme ou d'un genre. En 1583 donc, paraissent Les Appréhensions spirituelles, Poèmes et autres ouvres philosophiques : avec les recherches de la pierre philosophale, par F.B. de Verville, à M. du Guast, Paris, chez Timothée Jouan. Sous un intitulé qui déjà, distingue fallacieusement deux ouvrages, le recueil contient celui qui lui donne son titre Les appréhensions spirituelles ou entrée à la cognoissance des choses, discours auquel sont descriptes plusieurs raisons philosophiques ( $\left.\mathrm{f}^{\circ} 1-120\right)$ et un second, intitulé « recherches de la pierre " dont l'autonomie est signalée rétrospectivement par un explicit qui mentionne la « fin du premier livre des recherches philosophiques ». Ainsi désigné sous une forme unitaire, ce "premier livre » dont le titre ne correspond pas à celui du recueil (si bien qu'au sens strict, il n'existe pas) est lui-même composé de diverses pièces : il s'agit des « cognoissances necessaires, poème contenant plusieurs belles resolutions philosophiques", auquel s'ajoute « le livre de l'âme, où est faite une description entière de l'âme et de ses facultez »; mais ce « $\underline{\text { livre }}$ de l'âme " n'est lui-même que la troisième pièce des Appréhensions contenant, dans l'ordre, (1) les cognoissances nécessaires, 2) les stances de la mort et de la vie, à Pierre le Voirrier, 3 ) de l'âme et de ses facultés (fo25-47), 4) les dialogues de l'honneste amour et de la bonne grâce précédés et unifiés par une dédicace, 5) « Du bien de la mort commune », 6) Les recherches de la pierre Philosophale, traité où sont déduits plusieurs beaux points de la nature des métaux.

Pour compliquer l'inventaire, le titre de ce dernier " traité » change dans la table des matières : les " $\underline{\text { Recherches }}$ " y deviennent « $\underline{\text { Recherches de la pierre }}$ Philosophale et du moyen qu'il y faut tenir, si elle existe ou peut exister : avec une préface contre les soufleurs imposteurs et sophistes et quelques sentances

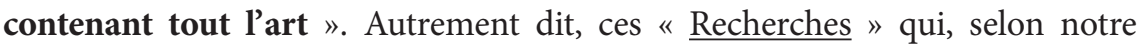
convention ne méritent pas d'italiques, imitent par leur distribution le schéma éditorial d'un ouvrage autonome : d'abord, des pièces liminaires, puis (fo 81-91) une "préface touchant les impostures qui se commettent en la recherche de la pierre Philosophale », ensuite (fo $91-120)$ « de la philosophie » dont l'explicit est signalé au $\left(\mathrm{f}^{\circ} 120 \mathrm{v}^{\circ}\right)$ par la mention citée plus haut : « fin du premier livre des recherches philosophiques ". Ce premier livre n'existe pas, on l'a dit. Mais y en aura-t-il un deuxième qui du même coup légitimerait la virtualité du premier? 
L'auteur lui-même pouvait l'espérer en 1583 ; disons tout de suite que ce n'est pas le cas, sinon à titre virtuel, on y reviendra.

Par ailleurs on mesure le rôle de la distribution des pièces dans l'effet qui les démultiplie. Le glossaire du paratexte est particulièrement fourni : "poème, œuvre philosophique, discours, livre, résolutions, traité, sentances "(sic). Chacun de ces termes sous-entend la pluralité des œuvres qu'ils désignent. Quant à la disposition, elle n' est pas moins éloquente : en introduisant au milieu du recueil des pièces "liminaires ", ou une préface, elle divise et distingue ce qu'inversement elle " unifie » par une dédicace, les deux procédés étant complémentaires ${ }^{7}$. Fragmentation, enchâssement et auto-référence sont ainsi les procédés purement éditoriaux qui inventent des œuvres souvent réduites à des espérances.

Et ce n'est pas tout, car le recueil ne s'arrête pas là : un complément de 15 folios (121-135) prétend lui adjoindre une " Muse céleste ou l'amour divin, contenant plusieurs sonets et stances à l'honneur de Dieu ». Ce nouveau titre (pas encore en italique selon notre convention) désigne un ensemble qui, augmenté de poèmes, parmi lesquels " de l'âme et de ses excellences » (à ne pas confondre avec " de l'âme et des ses facultés ") deviendra autonome en 1593, date à laquelle il sera réédité sous le titre La Muse céleste de Béroalde de Verville, (Tours : Jamet Mettayer, 1593).

Enfin cette addition n'est pas la dernière : à la suite du volume - mais il s'agit cette fois d'un effet qui ne tient qu'à la reliure, car le foliotage reprend au début - on trouve un autre recueil : Les soupirs amoureux de F. B. de Verville (Paris : Timothée Jouan, 1584). Il en existe une édition séparée (Paris : Timothée Jouan, 1583) qui sera reprise et augmentée en 1596, sous le titre : Les soupirs amoureux de F. B. de Verville : avec un discours satirique de ceux qui escrivent d'Amour par N. le Digne (Rouen Du Petit Val). Elle comprendra alors des pièces liminaires, puis 1) ( $\left.\mathrm{f}^{\circ} 3-48\right)$ Les souspirs amoureux proprement dits, 2) le discours satirique de Nicolas Le Digne à F.B. de Verville, 3) un ensemble de « poèmes libertins » parmi lesquels le célèbre « alchimiste » construit sur une équivoque scabreuse, (f $\left.\left.{ }^{\circ} 55-58\right), 4\right)$ des sonnets adressés à diverses « dames ».

Ainsi les pièces des recueils de 1583 apparaissent-elles rétrospectivement comme les " noyaux » d'œuvres futures qui, au fil d'une dérive scripturale et éditoriale, par détachement et augmentation des textes qui les composent, deviendront des œuvres autonomes, c'est-à-dire des livres. Encore fautil distinguer parmi ces virtualités les promesses tenues (La Muse céleste, 
Les souspirs amoureux) de celles qui ne le seront pas (le deuxième livre des " recherches philosophiques »). C'est impossible en 1583. L'effet d'annonce voulu par l'auteur ignore naturellement la différence entre réel et virtuel. C'est ainsi qu'une bibliographie se peuple de fantômes. Ceux des pièces revendiquées par l'auteur et auxquelles des bibliographes trop consciencieux et parfois facétieux, auront prêté le statut de livres.

\section{Un «fantôme " : le " deuxième livre des Recherches de la Pierre "}

On ne lui accorde pas d'italiques. C'est dire par avance le résultat de l'enquête. Mais alors qu'il est promis depuis 1583 dans le recueil des Appréhensions, cet hypothétique " deuxième livre des recherches de la pierre » jamais paru, fut-il au moins rédigé ? Il pourrait alors se confondre avec une autre œuvre, ou bien se déguiser sous un intitulé différent. Parmi les candidats figure un traité perdu intitulé de «l'art de la grande science sensuelle». Mais nul ne saurait jurer que ce second opus ait une existence autre que virtuelle. Certes, il est signalé par l'auteur dans son Palais des curieux (achevé d'imprimé du 5 novembre 1611) :

\section{(...) en mon œuvre de l'Art de la grande science sensuelle, je demonstre combien il y a és substances de sortes de liqueurs, de sels \& de terres : \& les moyens de les extraire parfaictement. ${ }^{8}$}

Et ce propos correspond effectivement à la " recherche de la pierre " mais on n'en a pas d'autre trace que cette allégation qui ne suffit pas à prouver son existence. À la quête alchimique, se superpose donc celle du « deuxième livre » alchimique de Verville. Voyons-en les étapes.

Cet hypothétique " deuxième livre » pourrait-il être un autre ouvrage, non moins hypothétique, intitulé les « sainctes résolutions" ? Cette autre œuvre perdue, à ne pas confondre avec les « résolutions philosophiques " contenues dans les " cognoissances nécessaires ", concernerait aussi le mouvement des astres (il s'agirait donc plutôt de " saintes révolutions ») : c'est du moins ce que Luzel croit pouvoir déduire de deux mentions fort elliptiques. L'une figure dans le Palais des curieux: 
(...) il ne faut pas estimer que le ciel qu'il nomme le troisiesme soit celuy qu'aux sphères nous mettons en tel ordre (...) il convient en penser autrement $\&$ de la sorte que nous avons faict en nos sainctes résolutions. ${ }^{9}$

L'autre, très antérieure, et qui date l'ouvrage d'avant le recueil des Appréhensions, lui permet de décrire les "saintes résolutions » comme une œuvre « certainement publiée à Genève avant $1579 »^{10}$. Il s'agit d'une pièce ajoutée au Blason des armoiries de Jérôme Bara, paru à Genève (Jean de Laon, 1579) et à Lyon (Barthelemy Vincent, 1581). Elle est signée des initiales P.M. accompagnées de la devise patientia omnia ducit - Luzel l'attribue à Pierre Manson - et elle concerne Verville qui a collaboré à la première édition :

Ainsi que cet auteur recherchoit curieux

Par l'art industrieux de la philosophie

Du naturel caché, la plus nette partie

De la terre et de l'eau et ce qu'ils ont de mieux

Que jadis figuroit le ruisseau gracieux,

Le cahos embrouillé, le Dragon oste-vie

La meurtrière poison, son amère ennemie,

Le rainceau du destin et le surnom des cieux.

Le temps qui semble avoir dessus nos faits envie

Le voulut empescher par ce trait d'armoirie

Pensant de sa recerche enfin le desgouter

Mais il s'est bien trompé (....). ${ }^{11}$

On y apprend, si on a bien lu, que Verville est lui-même cet auteur « curieux de la philosophie » / il s'agit d'alchimie / et qui ne s'en serait pas laissé détourner par ce "trait d'armoirie " (le commentaire des Blasons dont Bara n'aurait fourni que les images). Sa compétence en matière d'alchimie nous est signifiée par une figure énigmatique dont il serait l'auteur, et dont Luzel souligne un syntagme " surnom des cieux " parce qu' elle croit y voir une allusion aux prétendues « saintes résolutions ». Or nous croyons qu'elle se trompe. Car c'est l'ensemble des vers qu'il faut considérer. Il y est bien question d'alchimie et non d'astronomie. Mais il s'agit là d'une description parfaitement reconnaissable, quoique sommaire, du frontispice " alchimique " gravé par Thomas de Leu pour l'édition du Tableau des riches inventions Couvertes $d u$ voile des feintes 
Amoureuses qui sont représentées dans le songe de Poliphile, Dévoilées des ombres du songe \& subtilement exposées par Beroalde de Verville. On peut s'en assurer en vérifiant que les même emblèmes (le chaos, les dragons, le poison, le rainceau du destin) sont mentionnés dans le " commentaire steganographique » ajouté en tête de l'édition du Songe de Poliphile de Verville. C'est bien le cas, ce qui constitue une nouvelle énigme. Car cette édition et a fortiori son frontispice ne paraîtront qu'en 1600. De sorte qu'on aura progressé à rebours en faisant paraître avant sa naissance, le fantôme d'une œuvre " alchimique ", dans un contexte fictionnel, celui de l'interprétation du Songe de Poliphile. C'est le prétexte d'une autre forgerie, celle qui déguise ce dernier ouvrage par un titre inapproprié. On y reviendra donc.

Passons ensuite sur les étapes intermédiaires de l'enquête, pour n'en considérer que la fin. Entres autres fantômes attribués à Verville, on trouve, dans la Bibliotheca chemica de Pierre Borel la mention de Trios musicaux également disparus ${ }^{12}$. Il est peu probable qu'il s'agisse du " grand œuvre » annoncé. Mais il existe un candidat plus sérieux et plus tangible, quoique perdu lui aussi. Le testament de Verville, retrouvé par Idelette Ardouin-Weiss et Pierre Aquilon ${ }^{13}$ fait état d'un « exemplaire escript par lui faict et compillé intitulé de la phisicque ». L'auteur y demande qu'il soit «mis es mains desdits Anciens et surveillans de la religion prétendue refformée de ceste ville / Tours / pour estre par eulx s'il se trouve que bon soyt faict imprimer ». Et l'inventaire après décès décrit « ung cahier relyé non couvert contenant quatre vingt feuillez de papier escritz à la main, intitulé en ces motz le grand œuvre qui est l'introduction de la vraye congnoissance de la nature, annoté en plusieurs endroictz des marges de chacune page de la main dudit deffunct sieur de Béroalde ». La formule est ambiguë : faut-il comprendre que l'ensemble est de la main de Verville, ou seulement les annotations ? Quoi qu'il en soit, ce cahier, remis à un nommé Charles Houdry, le 4 janvier 1627, a disparu et son contenu n'a jamais été édité. À supposer que ce "grand œuvre qui est l'introduction de la vraye congnoissance de la nature " soit une ouvre originale, qu'elle se confonde (ou non) avec la compilation (?) intitulée " de la phisicque » - nul ne peut en juger - on peut légitimement présumer qu'il s'agit bien du projet du « deuxième livre de la recherche ». Un projet malchanceux : resté virtuel pendant plus de quatre décennies, il se sera finalement perdu.

On doute que l'histoire des sciences en ait été changée et ce n'est d'ailleurs pas ce qui nous importe, mais le fait qu'une œuvre, qu'elle soit virtuelle ou réelle, 
s'invente à travers des références disposées pour faire croire à son existence. Or le cas n'est pas isolé ; on peut ainsi étendre notre enquête à l'ensemble des procédés qui dans la bibliographie vervilienne, fabriquent des fantômes.

\section{La fabrique des fantômes : autour du Tableau des riches inventions}

En laissant de côté le cas particulier des pièces mentionnées dans le testament, la proportion des œuvres absentes prêtées à Verville semble trop importante, on l'a dit, pour n'être pas aussi une donnée fictionnelle ${ }^{14}$. A-t-il écrit un Traité de la duplication du cube, donné comme «non-imprimé en 1584 » par La Croix du Maine ${ }^{15}$ et mentionné par Colletet ? Ainsi que des Élémens Méchaniques (toujours selon La Croix du Maine) ? C'est possible, puisqu'il y renvoie dans le Cabinet de Minerve et récidive dans le Palais des curieux ${ }^{16}$. Mais il est possible aussi que ces Éléments que La Croix du Maine mentionne comme " non imprimés ", soient des fragments de son commentaire du Théâtre des Instrumens mathématiques \& méchaniques de Jaques Besson Dauphinois, docte mathématicien, avec l'interprétation des Figures d'iceluy par François Beroald, (Lyon : Barthelemy Vincent, 1579) ${ }^{17}$. Ouvrons à ce propos une parenthèse : l'activité de Verville «éditeur » (au sens de commentateur) se confond volontiers avec son travail « d'auteur », titre dont il prend soin de ne pas se prévaloir dans ce dernier ouvrage :

Cependant, afin que quelqu'un ne pense que je (...) me pare des plumes d'autruy, je vous présente avec les figures des machines qui sont de l'auteur / Besson / la déclaration d'icelles, sans laquelle elles seroyent (...) comme un corps sans âme ${ }^{18}$

Les commentaires dont il enrichitce qui n'està l' origine qu'un recueil de planches, changent la physionomie de l'œuvre, et l'on croit pouvoir signaler comme une innovation dans l'histoire du Livre, le procédé qu'il invente pour rapporter ses textes aux planches déjà gravées en taille douce. Faute de pouvoir y ajouter les lettres qui réfèreraient à ses légendes, selon un usage qui remonte au moins au Champ Fleury de Tory (1529), Verville imagine et fait graver une planche « virtuelle », orientée selon les points cardinaux par une « ligne septentrionale » (en haut), méridionale (en bas), occidentale et orientale (à gauche et à droite) au centre de laquelle est reproduite une « échelle », ou « mesure divisée en vingt 
quatre parties ». Ce sont les linéaments d'une cartographie fictive des planches qui suivent. Car en se servant des coordonnées mentionnées dans le texte, il suffit de reporter cette " mesure " sur les gravures figurant les machines, pour repérer les pièces qui sont l'objet du commentaire. Ce détour n'est qu'apparent, car Verville usera d'un procédé analogue (le commentaire d'une planche constituant un frontispice) pour métamorphoser en " roman alchimique » son édition du Poliphile.

Pour revenir aux œuvres fictives, dans le registre romanesque cette foisci, dès lors qu'on sait que «l'Hermitage d'honneur » est le centre fictionnel d'un roman resté longtemps en suspens, le Voyage des princes fortunés, on n'est plus obligé de croire que c'est le titre d'un livre parce que Guillaume Colletet qui l'a «veu autrefois » le tient pour tel ${ }^{19}$. Pragmatisme qu'on recommande, car il a le mérite de dissiper bon nombre de fantômes. Reste alors le cas, étonnamment fréquent, des impostures :

Dans un ouvrage labyrinthique et minutieux, intitulé Nicolas Flamel sous investigation ${ }^{20}$, Claude Gagnon a consacré plusieurs chapitres à l'histoire d'un faux célèbre et dont l'inexistence n'est d'ailleurs pas absolument démontrée. Il s'agit des Aventures d'Ali-el-Moselan surnommé dans ses conquestes Slomnal Calife de tepisone au Pais de Sterplie, traduit de l'arabe de Rabi El Ulloe de deon (Paris, 1582, in $12^{\circ}$ ). Ce titre qui multiplie les anagrammes - il faudrait lire " Nicolas Flamel » pour "Slomnal calife », Pontoise pour « Tepisone », « à sept lieues de Paris » pour « au pays de Sterplie » enfin, "Béroalde de Verville » pour « Rabi El Ulloe de deon » - est bien dans la manière de Verville, grand amateur d'anagrammes. C'est là un parfait exemple d'illusion référentielle : car ce titre aura suffit à convaincre les bibliographes (Quérard, Lachèvre et même V-L. Saulnier qui se contente d'émettre des doutes ${ }^{21}$ ) de l' existence d'une œuvre qu'on aimerait beaucoup lire, mais qui n'est probablement qu'une invention due au talent de Barthélemy Mercier de Saint-Léger (1734-1799), le premier à en faire mention.

On féliciterait Claude Gagnon de sa perspicacité, si elle ne visait d'abord à créditer l'auteur, d'une autre forgerie plus ancienne et plus tangible puisque le texte en est consultable : il s'agit du Livre des figures hiéroglyphiques « traduit par Arnaud de la Chevalerie » (Paris : Veuve Guillemot, 1612), faussement attribué à Flamel, et prêté à Verville. Disons sans prendre parti de manière définitive, que l'hypothèse semble vraisemblable : l'éditeur du Livre des Figures est aussi celui du Tableau des riches inventions, ce qui constitue un comparant 
convaincant ; mais que la " preuve " exhibée, n'a pas convaincu la critique ${ }^{22}$. Elle repose en effet sur l'inexistence supposée d'Arnaud de la Chevalerie ${ }^{23}$ dont le nom serait un anagramme : sa transcription latine permet en effet d'obtenir (à deux lettres près) celui de Béroalde de Verville ${ }^{24}$. Bref, en l'état actuel de la recherche, l'ouvrage n'appartient donc pas (ou pas encore) au corpus vervilien.

Enfin une mention moderne et parfaitement fictionnelle est le plus récent exemple de contrefaçon aux dépens de Verville. Elle figure dans La Vie mode d'emploi de Georges Pérec. L'auteur y insère, sous le titre de Tableau des riches inventions, une citation qui à l'évidence ne provient pas de cet ouvrage ${ }^{25}$. On n'ose dire qu'il s'agit-là d'une facétie, car c'est la description clinique d'un cadavre qu'à la lecture de W. ou un souvenir d'enfance, on présume être celui de la propre mère de Pérec, disparue dans les camps de concentration. Authentique fantôme donc, manifesté par l'artifice d'une fausse référence à une œuvre, le Songe de Poliphile, pour laquelle les oulipiens auront manifesté une constante prédilection ${ }^{26}$. C'est sans doute parce que sa version vervilienne est le prototype le plus accompli des contrefaçons prêtées à notre auteur. Il nous faut donc l'examiner aussi.

Pour l'avoir fait jadis ${ }^{27}$, en donnant l'inventaire des variantes d'un texte dont Verville, « éditeur » — répondant à la commande de Mathieu Guillemot ${ }^{28}$ - a effectivement révisé la traduction ${ }^{29}$, on voudrait revenir seulement sur l'artifice du paratexte. Celui-ci rappelle sous une forme plus sophistiquée, le procédé mis en œuvre dans son édition du Théâtre des machines. À un ouvrage dont il révise ou modifie le texte dans une proportion insignifiante, et qu'il ne commente nulle part (sinon, brièvement, dans une adresse au lecteur évasive et qui dément à la fois le titre et l'intention qu'il affiche, si bien que la mention « desvoilées des ombres du songe » est tout à fait fallacieuse) Verville se contente d'ajouter en tête, un "commentaire steganographique » du frontispice. Or, quoique nos confrères, trop prudents, n'osent l'affirmer, celui-ci n'a strictement aucun rapport avec l'intrigue et annonce tout autre chose, à savoir, les futurs romans « alchimiques» de notre auteur, lequel ne s'en cache d'ailleurs pas ${ }^{30}$ :

Quelques uns (...) voyant ce discours steganographique y donneront quelques moments de temps pour considérer la concurrence des esprits \& ainsi paistront leur curiosité en nos labeurs, qui bien tost Dieu aydant, vous produiront de nouvelles inventions qui satisferont une partie de vos désirs. 
Pourtant, cette intervention minimale aura suffi à faire croire au caractère « alchimique » de l'œuvre - au demeurant tout à fait innocente - et ainsi, à altérer durablement sa physionomie et sa réception. La part de Verville dans ce travestissement est difficile à évaluer. L'idée n'est pas de lui, mais de Jacques Gohory, qui dès 1554, dans l'avertissement de sa traduction du Livre XI de l'Amadis, a prêté un sens alchimique au Songe de Poliphile ${ }^{31}$. Toutefois la pièce liminaire de Pierre Manson citée plus haut, et qui réfère manifestement au frontispice de 1600 (ou à son ébauche) suggère une implication plus profonde. Si l'idée de la succession graphique du « cahos embrouillé », du « dragon » et du « rainceau du destin » est bien, dès 1579, de l'invention de Verville, celui-ci aura non seulement commenté mais aussi «programmé » le frontispice. Ce n'est pas étonnant. Ce qui l'est plus c'est que la lecture alchimisante, développée par Gohory en 1575 dans la préface du Quatorzieme livre de l'Amadis, qu'il traduit également, associait déjà la symbolique colonnienne, à celle de Nicolas Flamel, pseudo-auteur du Livre des Figures hiéroglyphiques:

Vous voyez icy (...) le dragon de Columna / l'auteur du Poliphile / \& sa caverne obscure, chose traictée pareillement par nos aucteurs françois (...) \& Nic./olas/ Flammel qui en a laissé de notables marques en tableaux à paris en divers temples, en dragons $\&$ anges de certaines couleurs. ${ }^{32}$

Gohory faisait allusion aux fameuses arches à décor " alchimique » du charnier des Innocents, dont le commentaire est justement l'objet du Livre des figures hiéroglyphiques ${ }^{33}$. Cette analogie entre le frontispice du Poliphile et l'iconographie du Livre des figures serait ainsi un argument en faveur de la thèse de Claude Gagnon, l'attribution à Verville de ce dernier ouvrage.

Quoi qu'il en soit, c'est le procédé qui nous importe. Son efficacité est redoutable. On se demande si, appliqué de nos jours à un ouvrage innocent par exemple le Tractatus logicus philosophicus de Wittgenstein, dont le titre et la matière se prêteraient bien à cette sorte de déguisement - il aurait le même succès et parviendrait à faire passer un pur logicien, ... pour un alchimiste. La comparaison est sans doute disproportionnée, mais elle donne une idée de la puissance de l'illusion référentielle qui nous occupe. Ce qui nous ramène au savoir-faire de Verville et au fond de notre propos : ce qu'on appellera la « fiction éditoriale » c'est-à-dire l' exploitation fictionnelle des usages éditoriaux. 


\section{La fiction éditoriale : de la Floride au Moyen de parvenir}

L'œuvre romanesque de Verville est le terrain privilégié d'une " fiction éditoriale " qui vise à confondre les œuvres et les genres. Dès l'origine, dans son cycle de la Floride ${ }^{34}$ qui paraît de 1592 à 1596, l'auteur multiplie les effets de brouillage. Les titres sont trompeurs : il n' est pas simple en effet de reconnaître, dans les titres des volumes successifs, le nom de l'héroïne. C'est que, selon un modèle hérité des romans d'aventures médiévaux, celle-ci (ainsi que la plupart des personnages) change constamment de nom et de condition, si bien que la « demoiselle " de la " première partie " devient "l'infante déterminée " de la " quatrième ». Dans le contexte du déguisement des œuvres de fiction, constante éditoriale du XVI ${ }^{e}$ siècle, la chose est presque banale.

En revanche, l'insertion entre les troisième et quatrième partie (selon la chronologie narrative) d'un recueil de "curiosités ", Le Cabinet de Minerve (Tours : Sebastien Molin, 1596, édition revue et augmentée : Rouen : Raphael du Petit-Val, 1601) distinct du roman, et étudié comme tel, quoiqu'il mette en scène ses personnages, nous semble un progrès décisif en ce qu'il déplace le jeu fictionnel et le transporte sur la scène éditoriale. L'énumération du titre fait valoir cette confusion parachevée par une indexation fictive des chapitres : Le cabinet de Minerve. Auquel sont plusieurs singularitez, Figures, tableaux, Antiques, Recherches saintes, Remarques sérieuses, Observations amoureuses, Subtilitez agréables, rencontres joyeuses \& quelques histoires meslées es avantures de la sage Fenisse, patron du devoir. Impossible de savoir si l'on a affaire à un « traité » ou à une œuvre de fiction et de deviner qu'il s'agit d'une extension de la Floride. Et même en ayant lu celle-ci et en sachant donc que Minerve n'est pas la déesse de la sagesse, mais un personnage du roman, le doute persiste, entretenu d'ailleurs par une critique qui persiste à étudier l'œuvre isolément.

Il est porté à son comble à la lecture du titre du Moyen de parvenir, le plus célèbre opus vervilien, le seul au demeurant dont notre auteur n'ait pas revendiqué la paternité : il la dénie ${ }^{35}$ au contraire dans son Palais des curieux rédigé en 1611, et c'est un excellent motif pour lui attribuer un ouvrage dont la première édition identifiée par Neil Kenny ne paraîtra qu'en 1616 ou 1617 (Paris : Veuve Guillemot ${ }^{36}$ ). À elle seule, cette œuvre aura justifié, dans les trois dernières décennies, la redécouverte de l'auteur : à juste titre, car le Moyen de parvenir, «CEuvre contenant (...) la raison de tout ce qui a esté, est, sera : avec démonstrations certaines et nécessaires selon la rencontre des effects de vertu 
(...) » n'est pas seulement l'opus magnum de Verville, mais aussi, l'éclatante illustration d'une conception étonnamment moderne de la fiction éditoriale.

Verville y met au service d'une théorie de la fiction, celle de la serendipité et de la « rencontre », l'imitation des nouveaux désordres de l'imprimé ${ }^{37}$ :

- Des feuillets et cahiers improprement assemblés produisent un texte discontinu, dont le narrateur constate le désordre en en rejetant la faute sur les hasards de la composition.

- Un discours découpé en « chapitres» dont les intitulés ("question, point, paraphrase, songe, proposition, couplet, cérémonie » mais aussi « risée » et « coïonnerie ${ }^{38}$ ) réfèrent aux procédés d'indexation et de découpage (voire à la vacuité de l'invention) plutôt qu' aux contenus.

- Des personnages, les quatre cents fantômes d'un immense dialogue des morts, parfois rendus anonymes par des désignations impersonnelles ("quelqu'un, cestuy là, un autre ...») qui font entendre leur voix en demeurant des fantômes - car jamais ils ne prennent corps autrement que par les discours qu'on leur prête, parfois in abstentia, lorsque leur entrée en scène n'est signalée qu'après qu'ils aient parlé.

L'ensemble illustre par avance la conception qu'expose Agamben de la virtualité de l'œuvre ${ }^{39}$ et, en ce sens, le Moyen de parvenir est bien, non pas un fantôme, mais l'ombre d'un livre impossible, défini selon sa propre formule, comme «le centre de tous les livres». Bien sûr la bibliographie rejoint sur ce point la critique littéraire. C'était inévitable car, perçu comme l'aboutissement de la trajectoire de l'œuvre entière, ce coup d'éclat explique et justifie l'abondance d'ouvrages virtuels dans la bibliographie vervilienne $\mathrm{e}^{40}$.

Revenons pour conclure, à la pure science bibliographique. Après avoir tantôt dissipé et tantôt étoffé les contours des fantômes verviliens, on voudrait en faire paraître un, aussi tangible qu'inattendu. Il s'agit d'une édition tardive $\mathrm{du}$ Tableau des riches inventions contenues dans le songe de Poliphile. Elle n'est à vrai dire pas entièrement inconnue, car Jean Theodore Graesse $\mathrm{e}^{41}$ en fait mention à l'entrée « Poliphilus » de son répertoire, où il signale l'édition « retouchée par Béroalde de Verville, Paris 1600 (ou avec un frontispice daté 1657)» mais sans lui accorder une attention particulière. Or l'existence de cet exemplaire, qu'on a pu consulter très brièvement dans la bibliothèque de la fondation Werner Oechslin 
à Einsiedeln, bien connue pour ses trésors dans le domaine de l'architecture, et qui est conservé sous la cote app. W3/12 920 - la référence est accessible en ligne dans l'inventaire des bibliothèques suisses - est à nos yeux un mystère. En effet, si l'on se fie à l'ouvrage de Henri-Jean Martin, Livre, pouvoirs et société à Paris au XVII siècle $^{42}$ ce Poliphile ne devrait pas exister. Sachant que son éditeur, Mathieu I Guillemot est décédé en 1610 et que l'inventaire de ses biens comprend « 13 bois pour le Songe de Poliphile » estimés à 10 livres tournoi, on doit conclure que l'ensemble a été démembré et dispersé, ce qui interdit toute impression ultérieure. Or, l'exemplaire de la bibliothèque Werner Oechslin (Paris : Pierre Aubouin, 1657) dépourvu du « commentaire steganographique » de Verville, peut-être jugé caduque (?), comporte les mêmes bois ${ }^{43}$ que l'édition Guillemot de 1600. Ils sont usés et la qualité du papier diffère de celle-ci, ce qui exclut en principe un tirage antérieur qui se confondrait avec celui de l'édition Guillemot. On ne s'explique donc pas l'existence d'un exemplaire qu'on a examiné trop brièvement, et il y a trop longtemps, pour être en mesure de le décrire plus avant. Mais on croit aussi qu'en matière de fantômes, c'est déjà avoir fait quelque chose que d'indiquer la référence d'un ouvrage qu'on livre ainsi à la curiosité des spécialistes, si d'aventure il s'en trouve pour se pencher sur lui.

\section{Notes}

1. Les participants au colloque « Bibliographie 2008 » organisé par Hélène Cazes à l'Université de Victoria, s'étonneront peut-être de ne pas trouver ici le texte de la communication qu'ils y auront entendue, consacrée à la « fabrication » des romans de la Renaissance (le Chevalier doré et Gérard d'Euphrate) publié séparément dans la revue Réforme, Humanisme, Renaissance ${ }^{\circ} 71$ (décembre 2010), p. 165-78). C'est que, selon le jugement d'Hélène Cazes, les lignes qui suivent et transcrivent notre communication à la session «bibliographie » du colloque de la SCÉR (Vancouver, juin 2008) ont semblé mieux s'intégrer au propos et à la méthode bibliographique de l'ensemble.

2. Véronique Luzel, "Le Palais des curieux de Béroalde de Verville, édition critique et commentée ", (thèse Université de Paris X-Nanterre, dir. Jean Céard, 2001). On renvoie tout particulièrement, à la recension bibliographique de l'introduction p. $96-110$. 
3. Giorgio Agamben, "Experimentum linguae, préface à l'édition française ", Enfance et histoire, destruction de l'expérience et origine de l'histoire, 1978, (Paris : PayotRivages, 2002 [c1978]), p. 7.

4. La bibliographie de Véronique Luzel (Le Palais des curieux de Béroalde de Verville, p. 96-110) ne numérote pas les pièces. Elle inclut, en tête des « œuvres de l'invention de Béroalde " un "Abrégé des œuvres de Cardan », qu’on rangerait plutôt parmi les traductions ou adaptations, d'autant qu'il est perdu et qu'on ne peut juger de son originalité. À ce décompte s'ajoute une traduction (de Colonna), un commentaire (celui de Besson) et une collaboration (au Blason des armoiries de Bara) soit environ 40 œuvres. Le contraste est éloquent si l'on s'avise que, selon les mêmes critères, la bibliographie établie par André Tournon à la fin du Cahier $V$-L. Saulnier consacré à l'auteur, n’en dénombre que 20 : A. Tournon, «Béroalde de Verville ", in N. Cazauran dir., Béroalde de Verville (Paris : Presses de l'École Normale Supérieure, coll. Cahiers V.-L. Saulnier 13, 1996) «Éléments d’une bibliographie» p. 213-21.

5. Agamben, "Experimentum linguae, préface à l'édition française ", Enfance et histoire..., p. 8.

6. Voir à ce sujet l'article d'Hélène Cazes, « La Flamme bibliographique : tradition et récits », dossier «Variations Bibliographiques », dans la revue @nalyses 6.2 (2011) : http://www.revue-analyses.org/index.php?id=1830

7. Comme l'a bien montré N. Kenny, dans son article « Car le nom mesme de libéralité sonne liberté : les contextes sociaux et économiques du savoir chez Béroalde de Verville ", dans N. Cazauran dir., Béroalde de Verville (Paris : École Normale Supérieure, coll. Cahiers V-L. Saulnier 13, 1996) p. 9, le procédé permet de multiplier les dédicataires. Il semble ainsi le strict équivalent de la pratique qui consistait à dédicacer les copies d'un manuscrit à différents mécènes.

8. Verville, Le Palais des curieux (1611), p. 510 : on cite d'après l'édition critique de Véronique Luzel, Le Palais des curieux de Béroalde de Verville.

9. Luzel, Le Palais des curieux de Béroalde de Verville, p. 229.

10. Luzel, Le Palais des curieux de Béroalde de Verville, p. 105.

11. On cite d'après l'édition : Jérôme de Bara, Livre des blasons (Lyon : Claude Ravot, 1579), p. 119.

12. Référence à Pierre Borel ?

13. I. Weiss et P. Aquilon « Béroalde de Verville : testament inédit et documents nouveaux », Bulletin de l'histoire du protestantisme français 132.4 (1986). 
14. Parmi les ouvrages « absents » qu’on propose de retirer provisoirement de la bibliographie vervilienne, figurent ceux que répertorie Luzel, soit : 1) « L'art de la grande science sensuelle » (signalé dans le palais des curieux, 2) Deux tragédies (sans nom, mentionnées dans les Appréhensions spirituelles, signalées par La Croix du Maine et Colletet) 3) «Le Dialogue de la mort » ou discours sur l'utilité de la mort (d'après Colletet) 4) «Le Dialogue de la vérité »(d'après La Croix du Maine) 5) « De la Duplication du cube » (d'après la Croix du Maine) 6) « Les elemens mechaniques » entre 1584 et 1611 (d'après La Croix du Maine, et Colletet) 7) "L'hermitage d'Honneur » (d'après Colletet) 8) «Les saintes résolutions », 9) Les «Trios musicaux» (selon Pierre Borel) 10) «Le second livre des recherches de la pierre philosophale », 11) « le voyage d'hercueil » joint à un volume des Appréhensions spirituelles d'après Viollet le Duc - mais ne s'agit-il pas du fameux opuscule de Ronsard ? - 12) «Le Conseil utile contre la famine avec un remède de santé pour les pauvres », enfin, 13) les improbables « aventures d'Ali - el-Moselan / Nicolas flamel/ surnommé dans ses conquêtes Slomnal Calife / Nicolas flamel/ de Tepisone /pontoise/ au pays de Sterplie » / à sept lieues de paris/traduit de l'arabe de rabi El Ulloe de deon / Beroalde de Verville/ Paris, (cf infra). En revanche il faudrait conserver « De la Phisicque ou le grand œuvre qui est l'introduction de la vraye congnoissance de la nature ", œuvre certes inédite et perdue, mais dont l'existence est attestée devant notaire.

15. Cf. V. Luzel, Le Palais des curieux de Béroalde de Verville, p. 102.

16. Le Palais des curieux, éd. Luzel, Le Palais des curieux de Béroalde de Verville, p. 551.

17. CEuvre de Jacques Besson, de conception ancienne puisque son privilège remonte au 27 juin 1569.

18. J. Besson, «Épître dédicatoire à M. François d'Hastings ", Theâtre des instrumens mathématiques (Bâle : s.e., 1578).

19. V. Luzel, Le Palais des curieux de Béroalde de Verville, p. 103.

20. Claude Gagnon, Nicolas Flamel sous investigation, suivi de l'édition annotée du Livre des Figures hiéroglyphiques (Québec : Le loup de gouttière, 1994).

21. L'importante étude de V.-L. Saulnier, «Études sur Béroalde de Verville, introduction à la lecture du Moyen de parvenir ", Bibliothèque d'humanisme et Renaissance 5 (1944), p. 209-326), fait encore, et à juste titre, autorité.

22. En particulier Didier Kahn, grand connaisseur en forgeries alchimiques, également éditeur du pseudo-Flamel : Nicholas Flamel, Écrits alchimiques, éd. Didier Kahn (Paris : Belles Lettres, 1993). 
23. Signalons que contrairement à ce que suppose Claude Gagnon (Nicolas Flamel sous investigation,), ce toponyme poitevin existe bel et bien : nous attestons avoir vu un manoir de ce nom en bas Poitou, dans le voisinage de la demeure et du jardin de William Christie.

24. La réfection en 1996 du volume du Dictionnaire des lettres françaises consacré au $\mathrm{XVII}^{\mathrm{e}}$ s (Patrick Dandrey dir., Dictionnaire des lettres françaises, vol. Le XVII ${ }^{\mathrm{e}}$ siècle [Paris : Fayard, 1996]) n'a pas pris en compte cette substitution; il est vrai que la notice consacrée à Arnaut de la Chevalerie ne souffle mot de l'inexistence du personnage.

25. Georges Pérec, La Vie mode d'emploi (Paris: Hachette, 1978), p. 343.

26. Rappelons qu'Albert-Marie Schmidt, fondateur avec Queneau, de l'Oulipo en a préfacé la réédition en facsimilé qui est toujours notre bible : Albert-Marie Schmidt, "Préface », in Francesco Colonna, Le songe de Poliphile, adap. Jean Martin, (Paris : Club des libraires de France, Paris, 1963).

27. Voir notre article «Les riches inventions de Béroalde de Verville », dans N. Cazauran dir., Béroalde de Verville, p. 111 et ss.

28. Verville le rappelle dans son avertissement : « il / Guillemot/ me l'a mit en main pour le revoir et faire parler plus poliment (...)».

29. Voir notre article, «Les riches inventions de Bérolade de Verville». Verville a limité ses interventions aux points stratégiques du livre (l'incipit, la rencontre de Polia au centre du Livre I et l'explicit) en concentrant ses variantes en haut et en bas de page, signe d'une révision superficielle et, par ailleurs, tout à fait fantaisiste.

30. On cite l'adresse non paginée, « aux beaux esprits qui arresteront leurs yeux sur ces projets de plaisirs sérieux ».

31. Lidée est attestée ensuite dans la préface du Demosterion de Roch le Baillif (Rennes : Pierre Le Bret, 1578), voir H. Baudry, « Noël du Fail préfacier du Demosterion ... », in Noël du Fail écrivain, éd. C. Magnien-Simonin (Paris : Vrin, 1991) p. 185-201.

32. Le Quatorzieme livre de l'Amadis, avertissement par Gohory (on cite d'après la postface de Didier Kahn à son édition du pseudo-Flamel, Écrits alchimiques, p. 105).

33. Voir notre article « L'effet de la bigarrure : les Contes et discours d'Eutrapel comme danse macabre », in M.-C. Thomine dir., Contes et discours bigarrés (Paris : Presses Universitaires Paris Sorbonne, coll. Cahiers V-L. Saulnier 28, 2011), p. 69-86.

34. La première partie paraît en 1592 à Tours, chez Jamet Mettayer, Floride, histoire françoise. En laquelle on peut voir les différents événemens d'Amour de Fortune et d'honneur et combien sont en fin agréablesles fruicts de la vertu (Tours : Jamet Met- 
tayer 1592) et revue et augmentée (Tours : Jamet Mettayer 1593) ; la dernière publication de 1596 circule sous le titre L'infante déterminée qui est la quatriesme partie des Avantures de Floride, où se voyent plusieurs trofées de la vertu triomfante du vice (Tours : Sebastien Molin, 1596). Ce titre a-t-il laissé croire à une œuvre distincte? C'est possible car ce volume est le seul de la série à n'avoir pas bénéficié d'une numérisation et d'une mise en ligne sur le site Gallica de la BNF.

35. Tout en l'avouant : « ce pendant je vous avise (...) que j'ay faict un œuvre, lequel est une Satyre universelle où je reprends les vices de chacun : je pensois vous le faire voir soubs un tiltre qui est tel, Le Moyen de parvenir, mais on me l'a vollé, si que pour en avoir le plaisir vous attendrez encor : je l'ay mis en tel estat que je l'avouray mien, au lieu que l'exemplaire dont on m'a fait tort est insolent \& que je dénierois estre de moy, aussi qu'il n'est pas de mon escriture, \& avec cela il n'est pas de mérite pour estre leu (...) » : Le Palais des curieux, objet LXI, éd. V. Luzel, p. 486-87. La mention de l'écriture, si elle ne réfère pas au style, laisse croire à la circulation manuscrite d'un exemplaire (au moins) du Moyen de parvenir, cinq ans avant ce qu'on croit être son édition princeps (cf. note suivante).

36. Cf. Neil Kenny, «Le Moyen de parvenir : the earliest known edition, its date, and the women who printed it ", Studies on Beroalde de Verville 17 (1992), p. 21-41. Voir cet ouvrage dans l'édition d'Hélène Moreau et André Tournon (Aix : Publications de l'Université de Provence, 1984), récemment reprise et amplifiée, avec la collaboration d'André Ristori (Paris : Champion, 2004), ainsi que l'édition folio Gallimard (Paris : Gallimard, 2006) par Michel Renaud, préfacée par Michel Jeanneret (je remercie tout particulièrement Michel Renaud qui m'en a fait parvenir un exemplaire).

37. Ce serait un là beau sujet de thèse (de l'École des Chartes ?) : étudier l'imitation ou l'exploitation des procédés liés à la fabrication matérielle du livre, dans la composition fictionnelle des œuvres littéraires du XVI ${ }^{\mathrm{e}}$ siècle.

38. Il s'agit respectivement des titres des chapitres 1 à 8 , puis 61 et 62 .

39. Giorgio Agamben, "Experimentum linguae », préface à l'édition française de Enfance et histoire, destruction de l'expérience et origine de l'histoire (Paris : Payot \& Rivages, 2002, p. 7).

40. L'objet LVI du Palais des curieux en donne cette justification : «(...) et puis que scait-on si tout ce qu'on dit est vray, \& si on n'a point changé les noms, comme on a veu de nouveaux mettre leurs noms aux anciens livres, dont ils recevoient de la gloire par ceux de leur siècle qui n'avoient point ouy parler de tels œuvres ? (...) 
la vérité du passé est tant brouillée qu'on n’y voit gueres clair (...) » V. Luzel, Le palais des curieux de Béroalde de Verville, p. 462.

41. Johann Georg Theodor Graesse, Trésor de livres rares et précieux ou nouveau dictionnaire bibliographique, (Genève : Slatkine, 1993 [Dresde : R. Kuntze, 1859-69]), tome V, p. 388.

42. Henri-Jean Martin, Livre, pouvoirs et société à Paris au XVII siècle, préface Roger Chartier (Genève : Droz, 1999), tome I, p. 383.

43. Rectifions au passage l'erreur de Henri-Jean Martin, qui fut aussi la nôtre; ces bois n’ont pas été « retaillés récemment » comme l'avait laissé croire Claude Popelin, éditeur d'une traduction française du Songe de Poliphile en 1883 (Paris : Liseux, réimpr. Genève, Slatkine, 1981), le remplacement de l'un des bois, celui de la structure de la porte de la pyramide, remonte en réalité à l'édition de 1554 . 